\title{
Caracterização de Pacientes com Feridas Neoplásicas
}

\section{Characterization of Patients with Neoplastic Wounds}

\author{
Caracterización de los Pacientes con Heridas Neoplásicas
}

Isabel Neves Duarte Lisboa ${ }^{1}$, Marilia Perrelli Valença ${ }^{2}$

\begin{abstract}
RESUMO
Objetivo: Identificar e discutir o perfil sociodemográfico e de saúde de pacientes que apresentam feridas neoplásicas. Métodos: Trata-se de um estudo quantitativo, transversal e observacional, aprovado pelo Comitê de Ética em Pesquisa, realizado no Hospital do Câncer de Pernambuco (HCP) no período de junho a agosto de 2013. O instrumento de coleta de dados abordou informações clínicas e epidemiológicas do paciente, dados específicos da lesão e do tratamento tópico. Resultados: Participaram do estudo 51 pacientes com ferida neoplásica; dentre eles, 60,8\% eram homens entre 40 e 60 anos. Conclusão: Os participantes apresentavam feridas localizadas, em sua maioria, na região da cabeça e do pescoço (52,8\%), apresentando estadiamento 3 em 52\% das lesões. Os produtos utilizados na instituição foram colagenase e ácidos graxos essenciais.
\end{abstract}

DESCRITORES: Estomaterapia. Serviço hospitalar de oncologia. Cicatrização. Qualidade de vida.

\begin{abstract}
Objective: Identify and discuss the profile sociodemographic and health of patients with neoplastic wounds. Methods: This is a quantitative, cross-sectional observational study, was approved by the Research Ethics Committee, held at the Hospital do Câncer de Pernambuco (HCP) in the period June to August 2013. The instrument for data collection addressed: epidemiological and clinical patient information, specific data of the lesion and topical treatment. Results: The study included 51 patients with neoplastic wounds, 60.8\% were men aged 40-60 years. Conclusion: Participants had wounds mostly located in the head and neck (52.8\%), with stage 3 in 52\% of lesions. The products used in the institution were collagenase and essential fatty acids.
\end{abstract}

DESCRIPTORS: Stomatherapy. Oncology sevice, hospital. Wound healing. Quality of life.

\section{RESUMEN}

Objetivo: Identificar y analizar el perfil sociodemográfico y la salud de los pacientes con heridas neoplásicas. Metodos: Se trata de un estudio observacional cuantitativo, transversal, fue aprobado por el Comité de Ética de la Investigación, que se celebró en el Hospital del Cáncer de Pernambuco (HCP) en el período de junio a agosto de 2013. El instrumento de recolección de datos abordar: la información del paciente epidemiológica y clínica, los datos específicos de la lesión y el tratamiento tópico. Resultados: El estudio incluyó a 51 pacientes con heridas neoplásicas, 60,8\% eran hombres de entre 40 a 60 años. Conclusión: Los participantes tenían heridas en su mayoría localizados en la cabeza y el cuello (52,8\%), con la etapa 3 en el 52\% de las lesiones. Los productos utilizados en la institución fueron colagenasa y ácidos grasos esenciales.

DESCRIPTORES: Estomaterapia. Servicio de oncología en hospital. Cicatrización de heridas. Calidad de vida.

'Enfermeira pela Universidade Federal do Rio Grande do Norte (UFRN). Pós-graduada em Cancerologia pela Universidade de Pernambuco (UPE) - Recife (PE) Brasil. Endereço para correspondência: Campus Universitário - Avenida Senador Salgado Filho, 3.000 - CEP 59078-970 - Lagoa Nova - Natal (RN), Brasil - E-mail: bebelisboa@gmail.com

Mestre em Ciências da Saúde pela Faculdade de Ciências Médicas da UPE - Recife (PE), Brasil.

*Artigo baseado em monografia apresentada em 19 de dezembro de 2013 no Hospital Universitário Oswaldo Cruz (HUOC/UPE).

Artigo recebido em: 27/10/2014 - Aceito para publicação em: 24/04/2015 


\section{INTRODUÇÃO}

Conhecido há muitos séculos, o câncer foi amplamente considerado como uma doença dos países desenvolvidos e com grandes recursos financeiros. Há aproximadamente quatro décadas, a situação vem mudando e a maior parte do ônus global do câncer pode ser observada em países em desenvolvimento, principalmente aqueles com poucos e médios recursos ${ }^{1}$.

As causas de câncer são variadas, podendo ser externas ou internas ao organismo, estando ambas inter-relacionadas. As causas externas relacionam-se ao meio ambiente e aos hábitos ou costumes próprios de um ambiente social e cultural. As causas internas são, na maioria das vezes, geneticamente predeterminadas, estando ligadas à capacidade do organismo de se defender das agressões externas. Esses fatores causais podem interagir de várias formas, aumentando a probabilidade de transformações malignas nas células normais².

O tratamento oncológico é baseado em cinco pilares fundamentais: cirurgia, quimioterapia, radioterapia, imunoterapia e hormonioterapia. O diagnóstico correto e o estadiamento são atitudes clínicas fundamentais no processo de tomada de decisão. $\mathrm{O}$ tratamento pode ser de objetivo curativo; entretanto, mesmo com todos os avanços, não se pode dizer que essa doença seja sempre curável, e, nos casos mais avançados, o cuidado paliativo é a meta ${ }^{3,4}$.

As feridas neoplásicas são formadas pela infiltração das células malignas do tumor nas estruturas da pele. Nesse caso, ocorre a quebra da integridade do tegumento em decorrência da proliferação celular descontrolada que o processo de oncogênese induz, levando à formação de uma ferida evolutivamente exofítica ${ }^{3}$. Essas feridas podem ocorrer por extensão do tumor primário, por uma metástase, bem como pela implantação acidental de células na pele durante um procedimento cirúrgico ou diagnóstico, ou ainda pela invasão de linfonodos próximos ao tumor primário. São caracterizadas principalmente por progressão rápida e inviabilidade de cicatrização, hemorragias, odor fétido, exsudato abundante, alto risco para infecção, alto risco para miíase, presença de necrose tecidual, dor, prurido e agressão do tecido saudável perilesional ${ }^{4,5}$.

Não há um consenso sobre a nomenclatura dessas feridas. As denominações mais utilizadas são "feridas neoplásicas" ou "feridas tumorais". De acordo com o aspecto da ferida, podem ser utilizados os termos: "feridas ulcerativas malignas" (quando estão ulceradas e formam crateras rasas), "feridas fungosas malignas ulceradas" (união do aspecto vegetativo e partes ulceradas), "feridas fungosas malignas" (quando são semelhantes à couve-flor) ou ainda "feridas neoplásicas vegetantes". Além disso, elas também podem ser classificadas pelo seu estadiamento, de acordo com a evolução e as características de coloração, exsudato, odor, dor e invasão tecidual ${ }^{1,6,7}$.

É possível observar que o paciente com feridas neoplásicas ainda representa um desafio para o enfermeiro no que concerne ao manejo dos sinais e sintomas físicos e psicológicos que essas feridas impõem. Desse modo, a qualidade desses cuidados pode se tornar o fator mais significativo na determinação da qualidade de vida desses pacientes. Não obstante, existe a consciência da grande lacuna que permanece em relação à base concreta do conhecimento que gera esse cuidado, o qual, ao longo do tempo, estrutura-se sobre bases empíricas, desprovido de estudos clínicos específicos ${ }^{3}$.

Diante das dificuldades que envolvem a prática do cuidar de pacientes com feridas neoplásicas e na intenção da contínua melhoria da qualidade da assistência de enfermagem, este estudo teve como base a seguinte questão norteadora: quem são os pacientes com ferida neoplásica? Como estão sendo tratados atualmente na instituição?

Diante de tal reflexão, elencamos como objetivo deste estudo identificar e discutir o perfil sociodemográfico e de saúde de pacientes com feridas neoplásicas.

\section{MÉTODOS}

Trata-se de um estudo quantitativo, tipo corte transversal e observacional, realizado em pacientes com ferida neoplásica internados no Hospital do Câncer de Pernambuco (HCP).

$\mathrm{O}$ HCP é referência estadual em Oncologia. Está inserido no Sistema Único de Saúde (SUS) e é uma instituição de saúde sem fins lucrativos e de reconhecida utilidade pública. Seus serviços buscam prestar assistência médica especializada aos portadores de neoplasia, oferecendo tratamentos para os seguintes tipos de tumores: sólidos, de mama, pulmão, cabeça e pescoço, dos aparelhos digestivo e ginecológico, de próstata, ósseos e de partes moles, pele, dentre outros. A Oncologia Clínica do HCP proporciona assistência e tratamentos de quimioterapia, hormonioterapia, imunoterapia, suporte oncológico, cardiologia/unidade de terapia intensiva (UTI), hemoterapia e clínica médica. 
A amostra deste estudo se deu por conveniência e correspondeu a 51 pacientes acometidos por feridas neoplásicas que atenderam aos critérios de inclusão e exclusão.

Os critérios de inclusão foram: ter mais de 18 anos, possuir ferida neoplásica e concordar em participar da pesquisa, assinando o Termo de Consentimento Livre e Esclarecido (TCLE). Os critérios de exclusão foram: pacientes portadores de outras lesões não caracterizadas como neoplásicas e ter idade inferior a 18 anos. A coleta de dados se deu no período de junho a agosto de 2013 por meio de um instrumento de pesquisa composto por roteiro de entrevista com questões objetivas.

Os dados foram organizados em uma planilha do Microsoft Excel 2007 e tabulados em relação a variáveis sociodemográficas, características das feridas e tratamentos utilizados. Posteriormente, foi realizada a análise estatística utilizando o software STATA/SE 12.0.

O projeto de pesquisa foi aprovado pelo Comitê de Ética em Pesquisa do Hospital Universitário Oswaldo Cruz, sob o CAE no 13369213400005192. Os participantes foram orientados quanto aos objetivos do trabalho e concordaram em participar assinando o TCLE.

\section{RESULTADOS E DISCUSSÃO}

Do total de 51 indivíduos, 24 (47,1\%) possuíam câncer primário na região de cabeça e pescoço, seguido por câncer de mama, com 12 pessoas $(23,4 \%)$. Nesse caso, sugere-se que a região de cabeça e pescoço seja a responsável pelos maiores índices de ferida neoplásica. A maioria dos pacientes com câncer de cabeça e pescoço apresenta doença avançada ao diagnóstico. O prognóstico sombrio se deve, por vezes, ao diagnóstico tardio, o qual ocorre, aproximadamente, em 60\% dos pacientes ${ }^{8,9}$. Além disso, o carcinoma epidermoide apresenta-se como a histologia mais comum entre as neoplasias de cabeça e pescoço ${ }^{10}$, podendo tornar esse local o mais suscetível ao aparecimento de lesões neoplásicas, devido à sua proximidade com o tecido subcutâneo. $\mathrm{O}$ câncer de mama é considerado de bom prognóstico quando diagnosticado e tratado precocemente. Entretanto, o atraso no diagnóstico vem impedindo que as pacientes sejam beneficiadas pelos procedimentos terapêuticos que poderiam, de fato, reverter o curso clínico da doença $a^{11}$. Sugere-se que a localização tumoral adjacente à epiderme também possa predispor o tecido mamário ao desenvolvimento de lesões neoplásicas à medida que a patologia se torna avançada (Tabelas 1 e 2).

Trinta e um participantes $(60,8 \%)$ eram do sexo masculino, e 20, do feminino (39,2\%). Relaciona-se essa diferença a maior incidência de câncer de cabeça e pescoço como doença primária nesses indivíduos. No Brasil, a incidência desse câncer ultrapassa 20 por 100.000 habitantes e é mais comum em homens ${ }^{9}$. Todavia, evidencia-se o alcance de índices cada vez maiores entre a população feminina, diminuindo, portanto, essa diferença, uma vez que houve nos últimos anos algumas mudanças no estilo de vida e, consequentemente, nos hábitos relacionados ao consumo de álcool e tabaco por parte dessa população ${ }^{8}$. É importante ressaltar a incidência de câncer de mama em segundo lugar dentre a amostra, tornando a porcentagem relacionada ao sexo feminino ainda mais significativa.

Tabela 1. Perfil do paciente com ferida neoplásica.

\begin{tabular}{|c|c|c|}
\hline Variáveis & $\mathrm{n}$ & $\%$ \\
\hline \multicolumn{3}{|l|}{ Sexo } \\
\hline Masculino & 31 & 60,8 \\
\hline Feminino & 20 & 39,2 \\
\hline \multicolumn{3}{|l|}{ Idade (anos) } \\
\hline$<40$ & 4 & 7,8 \\
\hline $40-60$ & 30 & 58,9 \\
\hline$\geq 60$ & 17 & 33,3 \\
\hline \multicolumn{3}{|l|}{ Escolaridade } \\
\hline Analfabeto & 20 & 39,2 \\
\hline Ensino fundamental incompleto & 24 & 47,1 \\
\hline Ensino fundamental completo & 2 & 3,9 \\
\hline Ensino médio incompleto & 4 & 7,8 \\
\hline Ensino superior incompleto & 1 & 2,0 \\
\hline \multicolumn{3}{|l|}{ Comorbidades } \\
\hline $\mathrm{Sim}$ & 14 & 27,5 \\
\hline Não & 37 & 72,5 \\
\hline \multicolumn{3}{|l|}{ HAS } \\
\hline $\mathrm{Sim}$ & 13 & 25,5 \\
\hline Não & 38 & 74,5 \\
\hline \multicolumn{3}{|l|}{ Antecedentes pessoais } \\
\hline $\mathrm{Sim}$ & 38 & 74,5 \\
\hline Não & 13 & 25,5 \\
\hline \multicolumn{3}{|l|}{ Tabagismo } \\
\hline $\mathrm{Sim}$ & 34 & 89,5 \\
\hline Não & 4 & 10,5 \\
\hline \multicolumn{3}{|l|}{ Etilismo } \\
\hline $\mathrm{Sim}$ & 32 & 84,2 \\
\hline Não & 4 & 10,5 \\
\hline
\end{tabular}

HAS: hipertensão arterial sistêmica.

Fonte: HCP, 2013. 
Houve uma incidência maior de ferida neoplásica na faixa etária entre 40 e 60 anos de idade. Isso reflete também a incidência geral do acometimento do câncer no estudo em questão, tendo em vista o fato de serem patologias presentes em faixas etárias mais avançadas. Evidências epidemiológicas mostram que a incidência do câncer de cabeça e pescoço aumenta com a idade, assim como o câncer de mama, que tem maior acometimento em mulheres acima de 50 anos $^{8-11}$.

No quesito escolaridade, a maioria das pessoas possuía o ensino fundamental incompleto $(47,1 \%)$, seguida por analfabetos $(39,2 \%)$. Deduz-se que a baixa escolaridade reflete diretamente no acometimento da ferida neoplásica, visto que o paciente mais instruído tem melhores condições de esclarecimento quanto ao tratamento adequado. $\mathrm{O}$ baixo nível de instrução dos pacientes pode levar a um atraso na percepção da doença e na procura por assistência médica e, consequentemente, para o diagnóstico, diminuindo suas possibilidades terapêuticas 9 . A ferida tumoral surge em processos avançados de carcinogênese, tendo, assim, forte associação com o conhecimento deficiente do paciente.

Em relação aos antecedentes pessoais, do total de 51 pessoas, $38(74,5 \%)$ possuíam algum precedente. Dos 38, 34 (89,5\%) eram tabagistas, e 32 (84,2\%), etilistas. Esse dado reflete a suma relevância do consumo de tabaco e de álcool sobre a incidência do câncer. Além disso, sugere-se que tais hábitos também sejam determinantes para o desenvolvimento

Tabela 2. Características clínicas dos pacientes com ferida neoplásica.

\begin{tabular}{|c|c|c|}
\hline Variáveis & $\mathrm{n}$ & $\%$ \\
\hline \multicolumn{3}{|l|}{ Câncer primário } \\
\hline Cabeça e pescoço & 24 & 47,0 \\
\hline Mama & 12 & 23,4 \\
\hline Interrogado & 2 & 3,9 \\
\hline Linfoma & 3 & 5,9 \\
\hline Ovário & 1 & 2,0 \\
\hline Partes moles & 1 & 2,0 \\
\hline Pele & 2 & 3,9 \\
\hline Região perianal & 4 & 7,9 \\
\hline Sarcoma & 1 & 2,0 \\
\hline Útero & 1 & 2,0 \\
\hline \multicolumn{3}{|l|}{ Terapia antineoplásica } \\
\hline $\mathrm{Sim}$ & 32 & 62,7 \\
\hline Não & 19 & 37,3 \\
\hline \multicolumn{3}{|l|}{ Tipo de terapia } \\
\hline Quimioterapia & 24 & 75,0 \\
\hline Radioterapia & 20 & 62,5 \\
\hline Cirurgia & 13 & 40,6 \\
\hline
\end{tabular}

Fonte: HCP, 2013. e a progressão da ferida neoplásica. Sendo a cabeça e o pescoço as regiões de maior incidência neoplásica nesta pesquisa, é importante ressaltar que o álcool e o tabaco são os principais fatores de risco para o acometimento desse cân$\operatorname{cer}^{10}$. Além disso, o etilismo é um fator de risco importante no surgimento do câncer de mama ${ }^{11}$.

Das 51 pessoas entrevistadas, apenas 14 possuíam comorbidades. Desse contingente, $13(25,5 \%)$ eram hipertensos e apenas 1 indivíduo possuía diabetes mellitus. A ausência de comorbidades no paciente com ferida neoplásica nos chama a atenção, pois o câncer, apesar de ser uma doença que compromete e debilita o paciente das mais variadas formas, nem sempre se encontra associado a outras doenças, sejam elas transmissíveis ou não, como diabetes mellitus, hipertensão arterial e doenças virais.

Do total de 51 pacientes, 32 (62,7\%) já tinham sido submetidos a alguma terapia antineoplásica. Desse quantitativo, 24 (75\%) realizaram quimioterapia em algum momento, $20(62,5 \%)$ passaram por radioterapia, e $13(40,6 \%)$, por cirurgia. O câncer, em sua fase inicial, pode ser controlado e/ou curado por meio do tratamento cirúrgico, quando este for o tratamento indicado para o caso ${ }^{11}$. Considerando essas informações, sugere-se que grande parte dos pacientes já chega ao serviço médico em estágio avançado, fazendo-se necessária a associação com outras modalidades, como quimioterapia sistêmica ou radioterapia. Salienta-se aqui a porcentagem significativa de pacientes $(37,3 \%)$ que chegaram ao serviço com lesão sem o passado de terapêutica antineoplásica, reforçando o pensamento de que o paciente, em seu primeiro contato com o serviço,já se encontra em estágios avançados. A radioterapia e a quimioterapia também podem ser utilizadas na ferida neoplásica para controle do exsudato e do sangramento, reduzindo o tumor e diminuindo a dor ${ }^{5}$. Contudo, a lesão continuará a se desenvolver até que algum tratamento oncológico seja empreendido com sucesso. Isso implica dizer que também podem existir indivíduos na amostra que realizaram terapêutica quimioterápica ou radioterápica, porém com fins apenas paliativos (Tabelas 3 e 4).

Em relação à localização da lesão, das 51 pessoas, 27 (52,8\%) tinham lesão na região de cabeça e pescoço, seguida por região da mama, com 10 pessoas (19,6\%). As lesões neoplásicas podem surgir por extensão do tumor primário, por uma metástase, implantação acidental de células na pele durante um procedimento cirúrgico ou diagnóstico; além disso, também podem ocorrer por invasão de linfonodos próximos ao tumor primário ${ }^{5}$. Conclui-se que a ferida neoplásica pouco 
surge em decorrência de episódios metastáticos, visto que a localização da ferida praticamente se iguala com a localização da doença primária.

O estadiamento da lesão se apresentou predominantemente em grau 3 em 26 pacientes (52\%), seguido pelo estadiamento 4 , com 8 pacientes (16\%), de um total de 51 pacientes. O estadiamento 1 caracteriza-se por pele íntegra, tecido de coloração avermelhada ou violácea, nódulo visível e delimitado, assintomático. $\mathrm{O}$ estadiamento $1 \mathrm{n}$ constitui-se como uma ferida fechada ou com abertura superficial por orifício de drenagem de exsudato límpido, de coloração amarelada ou de aspecto purulento, tecido avermelhado ou violáceo, ferida seca ou úmida, dor ou prurido ocasionais e sem odor ${ }^{12,13}$.

O estadiamento 2 caracteriza-se por uma ferida aberta que envolve: derme e epiderme; ulcerações superficiais, por vezes, friáveis e sensíveis à manipulação; exsudato ausente ou em pouca quantidade (lesões secas ou úmidas), bem como intenso processo inflamatório ao redor da ferida. Por fim, destacamos dentre as características dor e odor ocasionais ${ }^{12,13}$.

O estadiamento 3 constitui-se como uma ferida espessa envolvendo o tecido subcutâneo. Possui profundidade regular, com

Tabela 3. Caracterização das lesões neoplásicas.

\begin{tabular}{|c|c|c|}
\hline Variáveis & $\mathrm{n}$ & $\%$ \\
\hline \multicolumn{3}{|l|}{ Localização da lesão } \\
\hline Abdome & 1 & 2,0 \\
\hline Cabeça e pescoço & 27 & 52,8 \\
\hline Mama & 10 & 19,6 \\
\hline Sacra & 1 & 2,0 \\
\hline Região perianal & 6 & 11,8 \\
\hline Região torácica & 6 & 11,8 \\
\hline \multicolumn{3}{|l|}{ Tamanho } \\
\hline$<5 \mathrm{~cm}$ & 16 & 31,4 \\
\hline Entre 5 e $10 \mathrm{~cm}$ & 13 & 25,5 \\
\hline$>10 \mathrm{~cm}$ & 22 & 43,1 \\
\hline \multicolumn{3}{|l|}{ Sintomas } \\
\hline $\mathrm{Sim}$ & 46 & 90,2 \\
\hline Não & 5 & 9,8 \\
\hline \multicolumn{3}{|l|}{ Tipos de sintomas } \\
\hline Dor & 41 & 89,1 \\
\hline Prurido & 22 & 47,8 \\
\hline Cefaleia & 10 & 21,7 \\
\hline \multicolumn{3}{|l|}{ Estadiamento } \\
\hline 1 & 2 & 4,0 \\
\hline 2 & 7 & 14,0 \\
\hline 3 & 26 & 52,0 \\
\hline 4 & 8 & 16,0 \\
\hline $1 \mathrm{n}$ & 7 & 14,0 \\
\hline
\end{tabular}

Fonte: HCP, 2013. saliência e formação irregular. É friável, ulcerada ou vegetativa, podendo apresentar tecido necrótico liquefeito ou sólido e aderido, com odor fétido e exsudato. Possui lesões satélites em risco de ruptura, tecido de coloração avermelhada ou violácea, porém o leito da ferida encontra-se predominantemente amarelado ${ }^{12,13}$.

No estadiamento 4 tem-se a invasão profunda de estruturas anatômicas. Destaca-se uma profundidade expressiva, onde, por vezes, não se visualiza o seu limite, sendo, em alguns casos, com exsudato abundante, odor fétido e dor. Possui tecido de coloração avermelhada ou violácea, porém o leito da ferida encontra-se predominantemente amarelado ${ }^{12,13}$.

Sugere-se que as lesões em estadiamento 1, 1n e 2 sejam menos frequentes pelo fato de serem passíveis de controle e regressão com terapêuticas, como quimioterapia e radioterapia, tendo em vista o fato de serem estágios iniciais de lesão. Sugere-se também que o estadiamento 4 estará mais

Tabela 4. Caracterização das lesões e do tratamento dos pacientes com feridas neoplásicas

\begin{tabular}{|c|c|c|}
\hline Variáveis & $\mathrm{n}$ & $\%$ \\
\hline \multicolumn{3}{|l|}{ Exsudato } \\
\hline $\mathrm{Sim}$ & 43 & 84,3 \\
\hline Não & 8 & 15,7 \\
\hline \multicolumn{3}{|l|}{ Tipo de exsudato } \\
\hline Purulento & 25 & 58,1 \\
\hline Sanguinolento & 24 & 55,8 \\
\hline \multicolumn{3}{|l|}{ Odor } \\
\hline $\mathrm{Sim}$ & 37 & 72,5 \\
\hline Não & 14 & 27,5 \\
\hline \multicolumn{3}{|l|}{ Odor } \\
\hline Grau 1 & 26 & 70,3 \\
\hline Grau 2 & 6 & 16,2 \\
\hline Grau 3 & 5 & 13,5 \\
\hline \multicolumn{3}{|l|}{ Coloração } \\
\hline Amarelado & 34 & 66,7 \\
\hline Avermelhado & 33 & 64,7 \\
\hline Violáceo & 5 & 9,8 \\
\hline Necrose & 9 & 17,6 \\
\hline \multicolumn{3}{|l|}{ Terapia tópica } \\
\hline $\mathrm{Sim}$ & 40 & 78,4 \\
\hline Não & 11 & 21,6 \\
\hline \multicolumn{3}{|l|}{ Tipo de terapia tópica } \\
\hline Soro fisiológico & 15 & 37,5 \\
\hline Colagenase & 13 & 32,5 \\
\hline Metronidazol & 1 & 2,5 \\
\hline $\mathrm{AGE}$ & 3 & 7,5 \\
\hline Sulfadiazina de prata & 4 & 10,0 \\
\hline Outras coberturas & 5 & 12,5 \\
\hline
\end{tabular}

AGE: ácidos graxos essenciais.

Fonte: HCP, 2013. 
presente em pacientes terminais em virtude de sua característica invasiva, atingindo outros órgãos em profundidade, sendo esse tipo menos encontrado também.

Em relação ao tamanho das feridas, a maioria apresentava-se maior do que $10 \mathrm{~cm}$ de comprimento (43,1\%), seguida por feridas com menos de $5 \mathrm{~cm}(31,4 \%)$. Observa-se que a invasão em planos mais profundos é uma característica mais presente na lesão neoplásica, já que a quantidade de lesões extensas e lesões pequenas não apresentou variação tão significativa. Foram encontradas lesões em comprimento extenso em grau 1, assim como lesões pequenas em grau 3 ou 4, porém com profundidade ou acometimento tecidual significativo.

Dos 51 pacientes, 46 (90,2\%) referiram alguma sintomatologia em relação à ferida. A dor estava presente em 41 indivíduos (89,1\%), seguida de prurido em 22 pessoas $(47,8 \%)$ e cefaleia em 10 participantes $(21,7 \%)$. Durante o seu processo de crescimento acelerado, o tumor pode exercer pressão e/ou invasão sobre estruturas e terminações nervosas, ocasionando dor pelo processo inflamatório que cursa com esse crescimento agressivo, ocorrendo a liberação de histaminas, responsáveis pelas frequentes queixas de prurido ao redor da ferida. A cefaleia mostra-se presente principalmente em pacientes com câncer de cabeça e pescoço, em que há envolvimento linfonodal ${ }^{7}$.

Em relação à exsudação da lesão, do total de 51, 43 pacientes $(84,3 \%)$ possuíam feridas exsudativas. Desses 43 , 25 pacientes $(58,1 \%)$ apresentavam exsudato purulento, e 24 $(55,8 \%)$, exsudato sanguinolento. $\mathrm{O}$ exsudato é atribuído à permeabilidade capilar aumentada no leito da ferida, como consequência da neovascularização débil do tumor e pela secreção adicional de fator de permeabilidade vascular pelo próprio tumor ${ }^{1}$. Associa-se a esse processo a formação adicional de exsudato decorrente do processo inflamatório adjacente. A monitoração do exsudato diminui o odor, aumenta o conforto e melhora a autoestima do paciente. $\mathrm{O}$ controle da infecção local e da quantidade de tecido necrótico pode diminuir o exsudato da ferida em alguns casos ${ }^{3}$.

Do total de lesões, 34 (66,7\%) possuíam coloração amarelada, e 33 (64,7\%), coloração avermelhada. A predominância das cores amarelada e avermelhada está relacionada às características teciduais de feridas de estadiamento 3, assim como à necrose em graus superiores, destacando a cor violácea, mais encontrada em graus iniciais de feridas (1 e 1n).

No que diz respeito ao odor, dos 51 pacientes, 37 $(72,5 \%)$ possuíam odor em sua ferida. Desses 37 , obtivemos 26 pacientes $(70,3 \%)$ com odor grau 1 , seguidos por 6 pessoas $(16,2 \%)$ com odor grau 2. O odor é uma característica importante na ferida tumoral, sendo encontrado principalmente em estadiamentos avançados. A infecção por micro-organismos anaeróbios e tecidos desvitalizados causa o odor fétido, o qual tem sido descrito como o responsável por desconforto, constrangimento e sofrimento, em decorrência da sensação de enojamento e isolamento social que imputa o paciente ${ }^{4-6}$. A variação entre graus também tenderá a variar pela quantidade de trocas de cobertura, higienização, quantidade de exsudação e cobertura adequada utilizada. O grau 1 é o odor sentido ao abrir o curativo; o grau 2 é sentido ao se aproximar do paciente sem abrir o curativo; o odor grau 3, definido como forte e/ou nauseante, é sentido no ambiente sem abrir o curativo ${ }^{12}$.

Das 51 pessoas, $40(78,4 \%)$ utilizavam algum tipo de terapia tópica. Dessas 40, 15 pessoas $(37,5 \%)$ utilizavam apenas soro fisiológico, seguidas por 13 pessoas (32,5\%) utilizando colagenase. Outras coberturas foram utilizadas em menor número, como metronidazol, sulfadiazina de prata e ácidos graxos essenciais (AGE). Os cuidados das lesões neoplásicas seguem os mesmos preceitos do curativo ideal: manter o meio úmido, evitar a aderência ao leito da lesão, evitar trauma durante a remoção, auxiliar no desbridamento do tecido necrótico, controlar a hemorragia, permitir as trocas gasosas, manter isolamento térmico, absorver e conter o exsudato, evitar irritação superficial, reestabelecer a simetria corpórea do paciente e ser esteticamente aceitável ${ }^{5}$. Todavia, as feridas neoplásicas possuem características peculiares que devem ser levadas em consideração no momento de se iniciar a conduta com o paciente. $\mathrm{O}$ objetivo é alcançar o controle de secreção, odor, sangramento, dor e prurido, e a meta é o curativo confortável, funcional e estético ${ }^{13}$. A conduta terapêutica deve ser ajustada às características da lesão, obedecendo aos princípios de cuidados com feridas. A meta principal dessas condutas deixa de ser a cicatrização - que é improvável - e passa a focar:

- $\quad$ o conforto do paciente com relação à ferida;

- a prevenção e o controle dos sintomas locais ${ }^{3-12}$.

Para o controle de sangramentos, podem ser utilizados: a aplicação de esponja hemostática (constituída à base de colágeno); a manipulação de cuidados com uso de irrigação com solução fisiológica (SF) 0,9\% ou água tratada ao retirar curativo anterior; a utilização de curativos não aderentes; a aplicação de adrenalina tópica diretamente sobre os pontos sangrantes; o uso de drogas coagulantes orais e radioterapia anti-hemorrágica ${ }^{5}$. 
No que concerne ao odor, estão indicados: o uso de curativo de carvão ativado (envolto em uma lâmina de gaze); hidróxido de alumínio; metronidazol sistêmico e/ou tópico ${ }^{2}$. Recomenda-se observar o padrão alimentar do paciente e elaborar um plano de cuidados que evite a troca de curativos próxima aos horários das refeições ${ }^{5}$.

Para o alívio da dor, é possível considerar: pré-analgesia pelo uso de dose de resgate; uso de anestésico tópico ao redor da ferida; aplicação de hidróxido de alumínio diretamente no sítio da lesão; manipulação cuidadosa do leito da ferida, evitando o esfregaço; revisão da analgesia sistêmica em curso; uso de radioterapia antiálgica ${ }^{5}$.

Para o controle do exsudato, podem ser utilizados curativos absortivos, como carvão ativado, alginato de cálcio e compressa/gaze como cobertura secundária. Em alguns casos, a falta de proteção à pele íntegra perilesional pode levar à irritação e, consequentemente, ao prurido. Atualmente, para o controle do prurido está indicada a aplicação de esteroides tópicos, como dexametasona creme a $0,1 \% \%^{5-13}$.

O soro fisiológico, quando utilizado exclusivamente, mantém a ferida úmida, favorece a formação do tecido de granulação, amolece os tecidos desvitalizados, estimula o desbridamento autolítico e absorve exsudato ${ }^{12}$. A conduta seria adequada para uma lesão passível de cicatrização. A colagenase atua como agente desbridante em lesões superficiais, promovendo a limpeza enzimática das áreas lesadas e retirando ou dissolvendo, enzimaticamente, necroses e $\operatorname{crostas}^{5-12}$. O desbridamento pode reduzir o odor e a exsudação presentes nessas feridas; todavia, o sangramento capilar comumente presente e as grandes massas de tecido necrótico contribuem para que essa seja uma prática evitada. É importante ressaltar que a conduta terapêutica para a ferida neoplásica não se centraliza na cicatrização, em que a colagenase seria um produto de maior utilidade.

A sulfadiazina de prata é um bactericida utilizado no tratamento de feridas com grande potencial de infecção generalizada, como queimaduras, úlceras varicosas e feridas cirúrgicas infectadas, e atua impedindo o aparecimento de uma vasta gama de bactérias e leveduras na pele danificada ${ }^{12-15}$. Não há dados na literatura que embasem o seu uso em feridas neoplásicas, visto que também se trata de um agente auxiliador na cicatrização, assim como os AGE, que age na hidratação preventiva, além de possuir propriedades emolientes que protegem a pele e auxiliam no processo de cicatrização de feridas.
O metronidazol é um antibiótico ativo contra bactérias anaeróbicas e protozoários, exercendo atividades antibacterianas, antiparasitárias e anti-helmínticas, sendo indicado para feridas neoplásicas com odor grau $3^{5,16,17}$.

É importante ressaltar que a instituição não possuía uma variedade grande de produtos, bem como um protocolo a ser seguido acerca do manejo de feridas neoplásicas.

\section{CONCLUSÃO}

Neste estudo foram avaliados 51 pacientes com feridas neoplásicas, a maioria era do sexo masculino, apresentando câncer primário e ferida, predominantemente, na região de cabeça e pescoço. Essa população tinha entre 40 e 60 anos de idade e possuía baixa escolaridade. A grande parte era constituída por tabagistas e etilistas, porém poucos indivíduos possuíam alguma comorbidade associada, sendo a hipertensão arterial a mais citada. A maioria dos pacientes já tinha sido submetida a alguma terapia antineoplásica, sendo a quimioterapia a mais realizada. As lesões encontravam-se, em sua maioria, no estadiamento 3, com mais de $10 \mathrm{~cm}$ de comprimento, exsudato predominantemente purulento e com odor grau 1. A cobertura mais utilizada foi a colagenase. A maioria dos pacientes relatava dor no local da lesão.

Este estudo possibilitou o conhecimento acerca do paciente com ferida neoplásica, seus aspectos epidemiológicos e o manejo da ferida. Caracterizar o paciente significa conhecer e analisar suas particularidades e os aspectos envolvidos em seu universo, possibilitando o planejamento de uma atenção individualizada e direcionada, premissa da qualidade da assistência de enfermagem.

Nos últimos anos, com a transição demográfica, ocorreram mudanças no envelhecimento da população, aumentando a prevalência de câncer e de outras patologias crônicas, o que torna este estudo relevante e exalta a necessidade constante de mais pesquisas acerca dos cuidados com o paciente com ferida neoplásica. Sendo o enfermeiro um membro ativo do processo de cuidar, é necessária a avaliação criteriosa do paciente com esse tipo de lesão e suas especificidades, o que determinará o cuidado adequado conforme as características clínicas de cada ferida, a fim de proporcionar qualidade de vida ao paciente. Conhecer mais - e melhor - , para atuar com foco, direcionamento e especificidade, gera melhorias no atendimento ao paciente. 
O conhecimento deficiente acerca do manejo das feridas neoplásicas ainda é uma realidade não só por parte do enfermeiro, mas da equipe multiprofissional como um todo, sendo esse tipo de ferida conduzida com base no empirismo e em conhecimentos de outros tipos de lesões. Recomenda-se, aqui, uma atualização e a capacitação contínua do profissional que está à frente do cuidado do paciente oncológico e, além disso, a valorização do enfermeiro especialista, melhorando, desse modo, a qualidade da assistência ao paciente que se encontra em processo de constante fragilidade.

Além da relevância para uma melhor assistência de enfermagem, espera-se que esta pesquisa possa subsidiar novos estudos, contribuindo para o enriquecimento científico da enfermagem oncológica.

\section{REFERÊNCIAS}

1. Instituto Nacional de Câncer (INCA). Incidência de câncer no Brasil. Rio de Janeiro: INCA; 2012.

2. Instituto Nacional de Câncer (INCA). Ações de enfermagem para o controle do câncer: uma proposta de integração ensino-serviço. $3^{\text {a }}$ ed. rev. Rio de Janeiro: INCA; 2008.

3. Firmino F. Pacientes portadores de feridas neoplásicas em Serviços de Cuidados Paliativos: contribuições para elaboração de protocolos de intervenções de enfermagem. Rev Bras Cancerol. 2005;51(4):347-59.

4. Academia Nacional de Cuidados Paliativos. Manual de cuidados paliativos. Rio de Janeiro: Diagraphic; 2009.

5. Santos CMC. Revisão sistemática sobre tratamentos tópicos de lesões vegetantes malignas [dissertação]. São Paulo: Escola de Enfermagem da Universidade de São Paulo; 2007.

6. Agra G, Fernandes MA, Platel ICS, Freire MEM. Cuidados Paliativos e Ferida Neoplásica. Rev Bras Cancerol. 2013;59(1):95-104.

7. Aguiar RM, Silva GRC. Os cuidados de enfermagem em feridas neoplásicas na assistência paliativa. Rev Hospital Universitário Pedro Ernesto. 2012;11(2):82-88.

8. Alvarenga LM, Ruiz MT, Pavarino-Bertelli EC, Ruback MJC, Maniglia JV, Goloni-Bertollo M. Avaliação epidemiológica de pacientes com câncer de cabeça e pescoço em um hospital universitário do noroeste do estado de São Paulo. Rev Bras Otorrinolaringol. 2008;74(1):68-73.

9. Dallagnol JC, Reksidler MA, Oliveira BV, Orlandi D, Silva RCA, Pedruzzi PAG. Perfil epidemiológico dos pacientes com câncer de cabeça e pescoço que evoluíram a óbito antes de receber tratamento. Rev Bras Cir Cabeça Pescoço. 2011;40(2):57-60.

10. Sociedade Brasileira de Oncologia Clínica. Manual de Condutas. Câncer de Cabeça e Pescoço. Belo Horizonte: Sociedade Brasileira de Oncologia Clínica; 2011.

11. Brasil. Instituto Nacional de Câncer (INCA). ABC do câncer: abordagens básicas para o controle do câncer/Instituto Nacional de Câncer. Rio de Janeiro: INCA; 2011.

12. Brasil. Instituto Nacional de Câncer (INCA). Tratamento e controle de feridas tumorais e úlceras por pressão no câncer avançado. Rio de Janeiro: INCA; 2009. 42 p. (Série Cuidados Paliativos)

13. Junior JF, Fuly PSC. Análise de associação entre feridas neoplásicas, odor e tratamento: estudo transversal. Rev Enferm UFPE (Online). 2014;8(8):2938-40.

14. Firmino F. Feridas Neoplásicas: Estadiamento e Controle dos Sinais e Sintomas. Prática Hospitalar (Online). 2005;42:59-62.

15. Gomes IP, Camargo TC. Feridas tumorais e cuidado de enfermagem: buscando evidências para o controle de sintomas. Rev Enferm UERJ. 2004;12:211-6.

16. Leite AC. Feridas Tumorais: cuidados de enfermagem. Rev Científica do Hospital Central do Exército do Rio de Janeiro. 2007;2(2):36-40.

17. Pinto KCMS, Silva RMP, Pereira AL, Ribeiro DB. Produtos Utilizados no Tratamento de Lesões Neoplásicas Cutâneas. Rev Estima. 2009;7(1):39-43. 